\section{Removal of tunneled-cuffed catheters in children: is it safe to leave the cuff?}

\section{Dear Editor,}

Long-term tunneled central venous catheters (CVCs) represent mainstay in treatment of chronically ill children, especially in cases of malignancies or chronic renal failure. These devices provide reliable avenues for performing dialysis and delivering prolonged courses of chemotherapy, nutritional support as well as antibiotics, fluids and blood components. They also allow routine blood samples without necessity of repeated, painful and indiscriminate venipuncture.

Guidelines and protocols for placement, use and management have been disseminated in medical literature over the last decades. However, there is still lack of evidence regarding removal of such devices; one of the unsolved issues is whether or not to remove the cuff during the procedure.

Although instructions for use included in most of product system inserts (Hickman/Broviac/Leonard, Groshong, Proline, etc.) state that if the cuff remains in the subcutaneous tissue it must be dissected out through a small incision utilizing local anesthesia, some authors $(1,2)$ reported higher risk of wound infection and permanent scarring related to cut-down dissection. As a matter of fact, they advocate traction removal of the device; retention of cuff may be eventually tolerated, since "the benefits of avoiding a cut-down outweigh the small risk of subsequent infection or extrusion of the cuff" (1).

In 2007, Lee (3) published the largest review of literature on complications occurring during and after elective procedure of long-term tunneled CVC removal asserting that once the catheter is pulled out without dissecting the cuff, it may remain as an innocuous foreign body within the subcutaneous tissue. However, non-negligible risk of chronic foreign-body reaction, as well as of recurrent infection of the site is reported. In addition, the cuff may interfere with diagnostic imaging, may be confused as a metastatic deposit and last but not least may result in being esthetically unacceptable. For these reasons the author concludes that when removing a CVC, leaving the cuff within the exit site must be discouraged (3).

Regarding pediatric population, we recently published a series of 194 consecutive patients treated for malignancies undergoing elective long-term CVC removal in a dedicated procedure room, according to institutional protocol (4). Dissection of the cuff was routinely performed as a part of protocol, in order to avoid complications related to retention as showed in Figure 1. Those cases occurred before the protocol was approved. As a matter of fact, infection or foreign-body reaction required further medical treatment, often resulting in surgical removal. Considering pre-existing prolonged and difficult medical history of those children and families, we strongly recommend dissecting the cuff while removing the catheter, in order to avoid such complications and unnecessary additional treatment to these patients. It

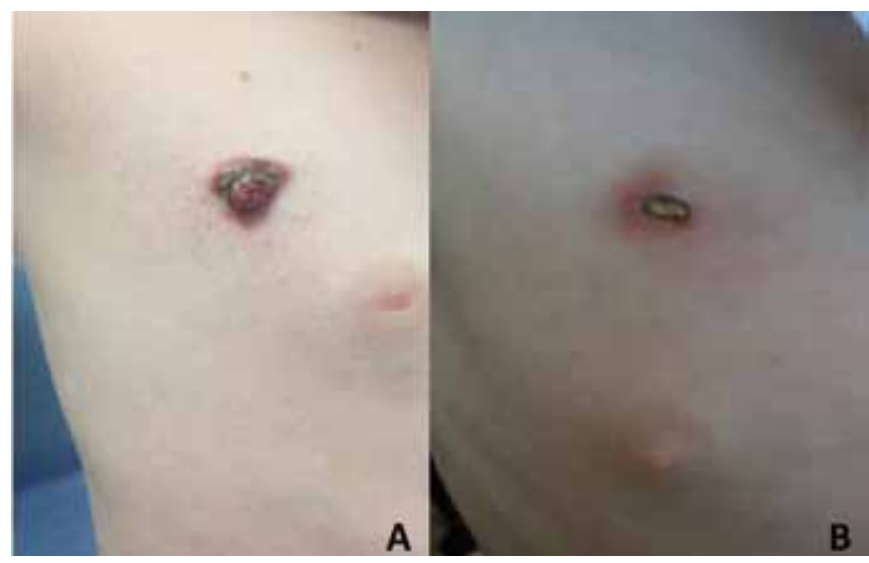

Fig. 1 - Retained catheter cuff as a cause of chronic inflammation. Subsequent foreign-body reaction and granuloma (A) as well as partial extrusion of the cuff (B) are also evident.

must be also borne in mind that subcutaneously anchored securement systems (SASS) have been recently approved for use in both adult and pediatric populations (5). These devices allow easier insertion fixation of the catheter, since the cuff is not required. For the same reason they also guarantee quick and simple removal, thus eliminating the issue of catheter cuff.

A randomized study is advocated to further corroborate these findings.

\section{Disclosures}

Financial support: None.

Conflict of interest: None.

\section{Alessandro Crocoli, Alessandra Narciso, Valerio Pardi, Antonella Accinni, Silvia Madafferi, Arianna Bertocchini, Alessandro Inserra}

General and Thoracic Surgery Unit, Bambino Gesù Children's Hospital IRCCS, Rome - Italy

\section{Corresponding author:}

Alessandro Crocoli

Unit of General and Thoracic Surgery

Bambino Gesù Children's Hospital, IRCCS

Piazza S. Onofrio 4

00165 Rome, Italy

alessandro.crocoli@opbg.net

\section{References}

1. Kohli MD, Trerotola SO, Namyslowski J, et al. Outcome of polyester cuff retention following traction removal of tunneled central venous catheters. Radiology. 2001;219(3):651-654.

2. Shibahara H, Shibahara N, Takahashi S. Cuff-related problems with a tunneled cuffed venous catheter. Ther Apher Dial. 2011;15(2):213-215. 
3. Lee AC. Elective removal of cuffed central venous catheters in children. Support Care Cancer. 2007;15(7):897-901.

4. Crocoli A, Martucci C, Leopardi E, et al. A dedicated protocol and environment for central venous catheter removal in pediatric patients affected by onco-hematological diseases. J Vasc Access. 2014;15(6):486-491.
5. Elen Hughes M. Reducing PICC migrations and improving patient outcomes. Br J Nurs. 2014;23(2):S12, S14-8.

Published online: January 31, 2017 